\title{
Perfil cardiovascular en pacientes con disfunción eréctil tratados en un centro de alta complejidad en la ciudad de Medellín, Colombia
}

\section{Cardiovascular Profile in Patients with Erectile Dysfunction Treated in a High Complexity Center in Medellín City, Colombia}

Juan Fernando Uribe Arcila ${ }^{1}$ Yerlin Andrés Colina Vargas²,3 Manuela Vélez Gaviria ${ }^{2}$ Laura Ceballos Naranjo ${ }^{4}$ Jonathan Cardona Vélez ${ }^{2,5}$ Jorge Hernando Donado Gómez ${ }^{4}$

${ }^{1}$ Médico Urólogo Especialista en Medicina Sexual, Hospital Pablo Tobón Uribe, Sociedad Latinoamericana de Medicina Sexual (SLAMS), Sociedad de Medicina Sexual de Norte América (SMSNA), Miembro del Consejo Superior Universidad CES, Medellín, Colombia 2 Universidad Pontificia Bolivariana, Medellín, Colombia

${ }^{3}$ Clínica Universitaria Bolivariana, Medellín, Colombia

${ }^{4}$ Facultad de Medicina, Universidad Pontificia Bolivariana, Medellín, Colombia

${ }^{5}$ Universidad de Antioquia, Hospital Universitario San Vicente Fundación, Medellín, Colombia

Urol Colomb 2020;29:148-152.
Address for correspondencia Yerlin Andrés Colina Vargas, MD, Clínica Universitaria Bolivariana, Universidad Pontificia Bolivariana, Carrera 72a,78B/50, Medellín, Antioquia, 13114, Colombia (e-mail: colinavargas1910@gmail.com).

\section{Resumen \\ Palabras clave \\ - disfunción eréctil \\ - enfermedades cardiovasculares \\ - enfermedad coronaria \\ - angina de pecho \\ - endotelio}

Objetivo Determinar el perfil cardiovascular de los pacientes con disfunción eréctil en un centro de alta complejidad de Medellín.

Métodos Estudio descriptivo, retrospectivo, en el que se incluyeron pacientes con diagnóstico de disfunción eréctil confirmado por el departamento de Urología, tratados en un centro de alta complejidad de Medellín entre 2010 y 2017; excluyendo aquellos con historia clínica con información incompleta o con desenlace cardiovascular previo al diagnóstico de disfunción eréctil. Los datos se obtuvieron de fuentes secundarias y se realizó su registro en una base de datos para su análisis mediante paquete estadístico (SPSS 24 Inc, Chicago, IL).

Resultados Se captaron, durante el periodo de estudio, 67 pacientes con disfunción eréctil que cumplieron los criterios de elegibilidad. Con una media de edad de 47,5 años. El $82 \%$ presentó disfunción eréctil severa, que estuvo asociada con el antecedente de angina, enfermedad coronaria y síndrome coronario agudo. Así mismo, más del $80 \%$ de los pacientes con diabetes, hipertensión, dislipidemia, tabaquismo, enfermedad renal crónica, obesidad y alcoholismo considerados como marcadores importantes de riesgo cardiovascular, presentaron disfunción eréctil severa.

Conclusión La comorbilidad cardiovascular en pacientes con DE es alta, existiendo una relación al compartir factores de riesgo y vías fisiopatológicas. Los pacientes con DE severa presentan mayor número de patologías asociadas, volviéndolos más propensos a desenlaces cardio-cerebrovasculares. received

July 18, 2019

accepted

October 14, 2019
DOI https://doi.org/ $10.1055 / \mathrm{s}-0039-3402484$. ISSN 0120-789X. eISSN 2027-0119.
Copyright $\odot$ 2020, Sociedad Colombiana License terms de Urología. Publicado por Thieme Revinter Publicações Ltda., Rio de Janeiro, Brazil. Todos los derechos reservados.

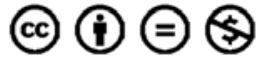




Abstract
Keywords
- erectile dysfunction
- cardiovascular
diseases
- coronary disease
- angina pectoris
- endothelium

Objective To determine the cardiovascular profile of patients with erectile dysfunction in a high complexity center in Medellín.

Methods Descriptive, retrospective study, in patients diagnosed with erectile dysfunction confirmed by the Department of Urology, treated in a high complexity center of Medellín between 2010 and 2017; excluding those with a clinical history with incomplete information or with a cardiovascular outcome prior to the diagnosis of erectile dysfunction. The data were obtained from secondary sources and their registration was made in a database for analysis by statistical package (SPSS 24 Inc, Chicago, IL).

Results 67 patients with erectile dysfunction were selected during the study period, who met the eligibility criteria. With an average age of 47.5 years. $82 \%$ had severe erectile dysfunction, and it was associated with a history of angina, coronary disease and acute coronary syndrome. Likewise, more than $80 \%$ of patients with diabetes, hypertension, dyslipidemia, smoking, chronic kidney disease, obesity and alcoholism, considered important markers of cardiovascular risk, presented severe erectile dysfunction.

Conclusion Cardiovascular comorbidity in patients with ED is high, there is a relationship, sharing risk factors and pathophysiological pathways. Patients with severe ED have a greater number of associated pathologies, making them more prone to cardio-cerebrovascular outcomes.

\section{Introducción}

La disfunción eréctil (DE) se define como la incapacidad para lograr o sostener una erección adecuada para completar una relación sexual. Es un trastorno común y su prevalencia aumenta con la edad, afecta $5 \%-10 \%$ de los mayores de 40 años, y entre $40 \%-70 \%$ de los mayores de 70 años; se estima que hay cerca de 150 millones de hombres afectados en todo el mundo, y para el 2025 afectará a más de 300 millones. $^{1-3}$

Muchos estudios han demostrado una correlación entre la DE y la enfermedad cardio-cerebrovascular (CCV), ambas comparten factores de riesgo como la edad, diabetes mellitus tipo 2 (DMt2), hipertensión arterial (HTA), dislipidemia, tabaquismo, obesidad, sedentarismo y el síndrome metabólico. ${ }^{4-6}$

Se cree que la DE y la enfermedad coronaria son manifestaciones clínicas del mismo proceso fisiopatológico, la disfunción endotelial severa. La DE representa un marcador temprano de enfermedad $\mathrm{CCV}$, así mismo en pacientes diabéticos, la DE es uno de los principales predictores de enfermedad coronaria. 6,7

El riesgo $C C V$ en los pacientes con DE, se estratifica en tres categorías que pueden servir para guiar el tratamiento. Riesgo bajo, corresponde a los pacientes sin un riesgo CCV importante relacionado con la actividad sexual, sin ser necesario que ellos se sometan a pruebas o evaluaciones cardiacas antes de reanudarla, ni que reciban tratamiento. Riesgo intermedio o indeterminado, incluye pacientes cuyo perfil de riesgo requiere pruebas y evaluación, previo a reanudar la actividad sexual; con los resultados de las pruebas se reclasifican en riesgo bajo o alto. Por último, alto riesgo, son quienes tienen una cardiopatía suficientemente grave 0 inestable para que la actividad sexual suponga un riesgo significativo, ellos deben someterse a evaluación cardíaca y tratamiento, y la actividad sexual debe interrumpirse hasta que la cardiopatía se estabilice con el tratamiento o el especialista decida que resulta seguro reanudarla. ${ }^{8-10}$

Los pacientes diagnosticados con esa condición, deben ser monitorizados en términos de prevención, diagnóstico temprano y tratamiento de los factores de riesgo para enfermedad CCV, con el fin de disminuir la mortalidad y morbilidad asociada a la misma. ${ }^{11,12}$ En el III consenso de Princeton, se indica que la función sexual, debe incorporarse en la evaluación de riesgo de enfermedad CCV. La evidencia sugiere que un enfoque integral para la reducción del riesgo, mejorará la salud vascular general incluida la función sexual. $^{13,14}$

De acuerdo con lo anterior, el objetivo del presente estudio fue determinar el perfil cardiovascular de los pacientes con disfunción eréctil en un centro de alta complejidad de Medellín.

\section{Métodos}

Se realizó un estudio descriptivo, retrospectivo, en el cual se incluyeron todos los pacientes con diagnóstico de $\mathrm{DE}$ tratados en un centro de alta complejidad de la ciudad de Medellín, entre el 2010 y el 2017. Para la obtención de la muestra se solicitaron la gestión de la información, los datos de la totalidad de pacientes a incluir filtrando mediante códigos CIE-10 específicos para DE, es decir, aquellos que corresponden a quienes se les confirmó el diagnóstico de DE 
por parte del servicio de urología de la institución, mediante el International Index Of Erectile Function - 5 (IIEF - 5), entre enero de 2010 y mayo de 2017. De acuerdo con dicha solicitud, se le entrega al equipo investigador un listado total de 83 pacientes de los cuales solo se ingresan 67 al estudio posterior a revisión de criterios de elegibilidad. Para ser incluidos, los pacientes debían tener diagnóstico de DE establecido por el departamento de urología; se excluyeron aquellos pacientes con historia clínica incompleta o quienes habían presentado desenlace cardiovascular mayor (enfermedad coronaria, enfermedad arterial oclusiva crónica, síndrome coronario agudo o enfermedad cerebro vascular/accidente isquémico transitorio), previo al diagnóstico de disfunción eréctil.

Adicionalmente con la finalidad de evaluar el perfil cardiovascular de los pacientes se tuvo en cuenta la presencia de: tabaquismo, dislipidemia, alcoholismo, HTA, obesidad, DMt2, enfermedad renal crónica, enfermedad coronaria, enfermedad arterial oclusiva crónica, síndrome coronario agudo, y, enfermedad cerebro vascular/accidente isquémico transitorio. $\mathrm{Y}$ adicionalmente paraclínicos tales como: creatinina, glicemia, HbA1C, perfil lipídico y TSH.

La severidad de la DE se clasificó según la escala de firmeza de la erección (The Erection Hardness Score (EHS)) así: 0 - El pene no se agranda. 1 - El pene es más grande, pero no duro. 2 - El pene es duro, pero no lo suficiente para la penetración. 3 - El pene es lo suficientemente duro para la penetración, pero no completamente duro, y 4 - El pene es completamente duro y completamente rígido. ${ }^{15}$

Se realizó un adecuado control de sesgos, incluyendo solo a los pacientes que cumplieran a cabalidad los criterios de selección, capacitando al equipo de investigación en la uniformidad de la recolección de los datos y consignando la información en una base previamente diseñada. Adicionalmente se evaluaron todas las posibles variables confusoras con el objeto de atribuir el riesgo a las principales variables tomadas en el estudio y no a las que pudieran causar un desenlace cardiovascular.

La información se obtuvo a partir de fuentes secundarias como la historia clínica electrónica de la institución y los resultados de los paraclínicos realizados durante el seguimiento, y se consignó sistemáticamente en una base de datos, donde posteriormente fue analizada en el paquete estadístico SPSS (SPSS 24 Inc, Chicago, IL). Las variables cualitativas se describieron mediante frecuencias absolutas y relativas y las cuantitativas se analizaron como media y desviación estándar o mediana y rango intercuartílico, según su distribución. El estudio fue aprobado por el Comité de Ética en Investigación institucional y los investigadores se adhirieron a la Declaración de Helsinki, versión 2013.

\section{Resultados}

Durante el periodo de estudio, se captaron 67 casos de pacientes con DE que cumplieron los criterios de elegibilidad; la media de edad fue de 47,5 años. El $82 \%$ de los pacientes tuvieron una DE severa; dicha severidad corresponde a la categoría 0 a 2 de la EHS. Las siguientes son las
Tabla 1 Comorbilidades de los pacientes con DE tratados en un centro de alta complejidad de Medellín, Colombia

\begin{tabular}{|l|l|}
\hline Comorbilidades & $\mathbf{n}(\%)$ \\
\hline Tabaquismo & $41(61,19)$ \\
\hline Dislipidemia & $41(61,19)$ \\
\hline Alcoholismo & $31(46,27)$ \\
\hline Hipertensión arterial & $31(46,27)$ \\
\hline Obesidad & $29(43,28)$ \\
\hline Diabetes mellitus 2 & $15(22,39)$ \\
\hline ERC* & $12(17,91)$ \\
\hline Enfermedad coronaria & $7(10,45)$ \\
\hline EAOC* & $5(7,46)$ \\
\hline SCA & $5(7,46)$ \\
\hline ECV/AIT* & $1(1,49)$ \\
\hline
\end{tabular}

Abreviaturas: EAOC, enfermedad arterial oclusiva crónica; ECV/AIT, enfermedad cerebro vascular/accidente isquémico transitorio; ERC, enfermedad renal crónica; SCA, síndrome coronario agudo.

comorbilidades de los pacientes en el estudio (- Tabla 1), de los cuales el 56,72\% tenían 3 o más; siendo las más comunes el tabaquismo y la dislipidemia con un $61,19 \%$, seguidas por la hipertensión arterial y el alcoholismo con un 46,27\%.

Los medicamentos más frecuentemente empleados dadas las enfermedades de base de los pacientes del estudio, fueron las estatinas en un $61,19 \%$, los IECA/ARAll en un $44,78 \%$ y los inhibidores de la fosfodiesterasa tipo 5 en un $37,31 \%$. En menor porcentaje estuvieron el ácido acetil salicílico 20,9\% y los betabloqueantes 8,96\%. Siendo menos usados los medicamentos naturales u homeopáticos para el tratamiento de la disfunción per se, que representaron solamente un 4,48\%.

De los 47 pacientes que contaron dentro de su historia clínica con glicemia en ayunas, 30 de ellos (44,78\%) tuvieron glicemias $<100 \mathrm{mg} / \mathrm{dL}$, el $11,94 \%$ tuvieron glicemias entre $100-125 \mathrm{mg} / \mathrm{dL}$ y finalmente el $13,43 \%$ valores mayores o igual a $126 \mathrm{mg} / \mathrm{dL}$ ( - Tabla 2).

Al correlacionar el antecedente de DM con la severidad de la $\mathrm{DE}$, se encontró que 14 de los 15 pacientes diabéticos tenían DE severa. Asimismo, se evidenció que 10 de los 12 pacientes con ERC y 28 de los 31 pacientes con HTA tuvieron

Tabla 2 Paraclínicos de los pacientes con DE tratados en un centro de alta complejidad de Medellín, Colombia

\begin{tabular}{|l|l|}
\hline Paraclínicos & Mediana $\left(\mathbf{P}_{25}-\mathbf{P}_{75}\right)$ \\
\hline Creatinina & $0,97(0,82-1,2)$ \\
\hline Glicemia & $92(85-120)$ \\
\hline HbA1C (\%) & $6,4(5,72-7,24)$ \\
\hline Colesterol total & $220(112-199,5)$ \\
\hline LDL & $129,5(108-170)$ \\
\hline HDL & $40(34-45)$ \\
\hline TSH & $2,3(1,88-3,64)$ \\
\hline
\end{tabular}


Tabla 3 Frecuencia de pacientes con DE severa en relación con las diferentes comorbilidades, en un centro de alta complejidad de Medellín, Colombia

\begin{tabular}{|l|l|}
\hline Comorbilidad & DE Severa n (\%) \\
\hline Enfermedad Coronaria & $7(100)$ \\
\hline Síndrome Coronario Agudo & $5(100)$ \\
\hline Diabetes Mellitus tipo 2 & $14(93,33)$ \\
\hline Hipertensión Arterial & $28(90,32)$ \\
\hline Dislipidemia & $37(90,24)$ \\
\hline Tabaquismo & $35(85,36)$ \\
\hline Enfermedad Renal Crónica & $10(83,33)$ \\
\hline Obesidad & $24(82,75)$ \\
\hline Alcoholismo & $25(80,64)$ \\
\hline
\end{tabular}

DE severa. La correlación de la gravedad de la DE con otras comorbilidades puede verse en la - Tabla 3 .

Desde el punto de vista cardiaco, todos los pacientes en el estudio con antecedente de enfermedad coronaria o aquellos que presentaron un SCA $(7,4 \%)$, tuvieron una mayor severidad de la enfermedad y de los 5 pacientes que cursaban con angina, 4 tuvieron DE severa. El promedio de años transcurridos entre el diagnóstico de la DE y el desarrollo de un SCA fue de 3,6, con un valor mínimo de 1 y un valor máximo de 6 años.

\section{Discusión}

Las enfermedades crónicas no transmisibles y en particular la enfermedad cardiovascular se ha posicionado como una de las más importantes causas de morbimortalidad a nivel internacional y nacional. Adicionalmente constituyen un problema de salud pública pues conllevan en sí mismas, altas tasas de discapacidad. ${ }^{16}$

La etiología de la enfermedad cardiaca y vascular es diversa, sin embargo, la mayoría de sus causas comparten un proceso fisiopatológico común y por lo tanto, tendrán un marcador que señala el inicio de una serie de eventos. En ese caso, el punto de partida al cual nos referimos es la DE, que ha sido a través de los años correlacionada con el desarrollo síndrome coronario agudo. ${ }^{5}$

En el presente estudio, la mayoría de los pacientes captados se encontraban en la quinta década de la vida, con una mediana de 48 años y en estudios anteriores, donde se evaluaron las principales características sociodemográficas y clínicas de los pacientes con DE, se encontraron datos similares donde las poblaciones iban desde los 40 a los 70 años, ${ }^{3}$ sin embargo, debe resaltarse que nuestro estudio encontró pacientes incluso en la tercera década de la vida lo cual podría considerarse una edad de inicio temprana si se tiene en cuenta la premisa de que ese es uno de los factores de riesgo más importantes para el desarrollo de la patología y que se referencia que el mayor porcentaje de la población con esa condición es mayor de 70 o incluso 80 años, puesto que el riesgo de presentarla a esa edad aumenta hasta en un $86 \%{ }^{17,18}$
La DE y la enfermedad coronaria comparten el mismo proceso fisiopatológico y por ende tienen entre ellas factores de riesgo similares. ${ }^{19}$ En nuestro estudio, las principales comorbilidades/factores de riesgo fueron el tabaquismo, la dislipidemia, la hipertensión arterial y el alcoholismo, lo cual se corresponde con la literatura actual donde la mayoría de los pacientes eran fumadores activos y consumidores de alcohol ${ }^{17-19}$. En otros estudios, se propone adicionalmente la presencia de enfermedad renal crónica como un factor de riesgo independiente para el desarrollo de DE, y se plantea que la severidad de la DE en conjunto con la presencia de ERC con una baja tasa de filtración glomerular, podría constituir un riesgo aumentado de presentar enfermedad coronaria. ${ }^{4}$

A pesar de que en los estudios encontrados se hace hincapié en la relación fisiopatológica que existe entre la DE y otras enfermedades del espectro del síndrome metabólico, tales como la diabetes, la hipertensión, la enfermedad renal crónica, etc, y se recalca que esa condición se presenta en los hombres antes de que aparezcan las demás manifestaciones del daño vascular, incluidos efectos de ateroesclerosis y enfermedad coronaria, ${ }^{8}$ se encuentran realmente pocos estudios a nivel local donde se relacione el síndrome coronario y la enfermedad vascular con la DE, por eso, la presente investigación se considera como de valor pues constituye una aproximación inicial al perfil sociodemográfico, clínico y paraclínico de los pacientes con DE en el contexto regional.

Aunque aún falta desarrollar otras investigaciones con un mayor tamaño poblacional y posiblemente con un seguimiento a largo plazo, se consideran nuestros resultados como concordantes con la literatura actual y por lo tanto, puede nuestra población beneficiarse de los adelantos futuros en materia de tamizaje y diagnóstico temprano del proceso de daño endotelial que se manifiesta como DE y que desembocara en un posible evento cardiovascular.

La presente investigación fue realizada con interés académico y formativo, en la cual se aclara que dentro de sus principales debilidades está el tamaño de la muestra, principalmente evidenciado por el poco registro de los CIE 10 de la patología en las historias clínicas, método por el cual se logran captar los pacientes en estudios retrospectivos con determinada condición.

\section{Conclusión}

Concordando con la literatura, el estudio muestra un aumento en la frecuencia de comorbilidad cardiovascular en los pacientes con DE, existiendo una relación al compartir factores de riesgo y vías fisiopatológicas. Al realizar esta investigación en un centro de alta complejidad, los pacientes presentaron un mayor número de patologías asociadas y ellas a su vez, fueron más prevalentes en quienes tenían diagnóstico de DE severa, dando como resultado, una mayor propensión a un desenlace CCV. Por lo descrito, la DE debe seguir considerándose como siendo un marcador temprano importante para dichos eventos. 
Nota

La investigación se desarrolló en el Hospital Pablo Tobón Uribe Medellín, Colombia.

Conflicto de intereses

Los autores declaran no tener ningún conflicto de intereses.

\section{Agradecimientos}

Al Hospital Pablo Tobón Uribe, por su aval para el desarrollo de la investigación en la institución y por las facilidades brindadas para el acceso a la información.

\section{Referencias}

1 Demirkol S, Balta S, Kucuk U, et al. Association between microvascular angina and erectile dsyfunction. Int J Impot Res 2014;26(04):124-127

2 Katsiki N, Wierzbicki AS, Mikhailidis DP. Erectile dysfunction and coronary heart disease. Curr Opin Cardiol 2015;30(04):416-421

3 Brock G. Diagnosing erectile dysfunction could save your patient's life. Can Urol Assoc J 2014;8(7-8, Suppl 5)S151-S152

4 Canat L, Canat M, Guner B, Gurbuz C, Caşkurlu T. Association between renal function, erectile function and coronary artery disease: detection with coronary angiography. Korean J Urol 2015;56(01):76-81

5 Sanchez E, Pastuszak AW, Khera M. Erectile dysfunction, metabolic syndrome, and cardiovascular risks: facts and controversies. Transl Androl Urol 2017;6(01):28-36

6 Ceballos MP, Álvarez Villarraga JD, Silva Herrera JM, Uribe JF, Mantilla D. Guía de disfunción eréctil. Sociedad Colombiana de Urología. Urol Colomb 2015;24(03):185.e1-185.e22

7 Gazzaruso C, Giordanetti S, De Amici E, et al. Relationship between erectile dysfunction and silent myocardial ischemia in apparently uncomplicated type 2 diabetic patients. Circulation 2004;110(01):22-26

8 Wespes E, Amar E, Eardley I, et al. Guía clínica sobre la disfunción sexual masculina: disfunción eréctil y eyaculación precoz. European Association of Urology; 2010:843-900
9 Wespes E, Amar E, Hatzichristou D, Montorsi F, Pryor J, Vardi Y; European Association of Urology. Guidelines on erectile dysfunction. Eur Urol 2002;41(01):1-5

10 Levine GN, Steinke EE, Bakaeen FG, et al; American Heart Association Council on Clinical Cardiology; Council on Cardiovascular Nursing; Council on Cardiovascular Surgery and Anesthesia; Council on Quality of Care and Outcomes Research. Sexual activity and cardiovascular disease: a scientific statement from the American Heart Association. Circulation 2012;125(08): 1058-1072

11 Menezes A, Artham S, Lavie CJ, Milani RV, O’Keefe J. Erectile dysfunction and cardiovascular disease. Postgrad Med 2011;123 (03):7-16

12 Pastuszak AW, Hyman DA, Yadav N, et al. Erectile dysfunction as a marker for cardiovascular disease diagnosis and intervention: a cost analysis. J Sex Med 2015;12(04):975-984

13 Montorsi P, Ravagnani PM, Galli S, et al. Association between erectile dysfunction and coronary artery disease. Role of coronary clinical presentation and extent of coronary vessels involvement: the COBRA trial. Eur Heart J 2006;27(22):2632-2639

14 Nehra A, Jackson G, Miner M, et al. The Princeton III Consensus recommendations for the management of erectile dysfunction and cardiovascular disease. Mayo Clin Proc 2012;87(08): 766-778

15 Mulhall JP, Goldstein I, Bushmakin AG, Cappelleri JC, Hvidsten K. Validation of the erection hardness score. J Sex Med 2007;4(06): 1626-1634

16 Enriquez DMB, Oyola KVL, Ruiz JEY. Cardiovascular Risk Factors and Diabetes in Medical Students: Observational Study. Exp Colomb 2018;6(02):61-65

17 Feldman HA, Johannes CB, Derby CA, et al. Erectile dysfunction and coronary risk factors: prospective results from the Massachusetts male aging study. Prev Med 2000;30(04): 328-338

18 Weber MF, Smith DP, O'Connell DL, et al. Risk factors for erectile dysfunction in a cohort of 108477 Australian men. Med J Aust 2013;199(02):107-111

19 Meller SM, Stilp E, Walker CN, Mena-Hurtado C. The link between vasculogenic erectile dysfunction, coronary artery disease, and peripheral artery disease: role of metabolic factors and endovascular therapy. J Invasive Cardiol 2013;25(06):313-319 